\title{
Solving the Multi-Period Water Distribution Network Design Problem with a Hybrid Simulated Anealling
}

\author{
Carlos Bermudez ${ }^{1}$, Carolina Salto ${ }^{1,2}$, and Gabriela Minetti ${ }^{1(凶)}$ \\ 1 Facultad de Ingeniería, Universidad Nacional de La Pampa, Calle 110 Nro. 390, \\ General Pico, La Pampa, Argentina \\ bermudezc@yahoo.com, \{saltoc, minettig\}@ing.unlpam.edu.ar \\ 2 CONICET, Buenos Aires, Argentina
}

\begin{abstract}
This work presents an optimization technique based on Simulated Annealing (SA) to solve the Water Distribution Network Design problem, considering multi-period restrictions with time varying demand patterns. The design optimization of this kind of networks is an important issue in modern cities, since a safe, adequate, and accessible supply of potable water is one of the basic necessities of any human being. Given the complexity of this problem, the SA is improved with a local search procedure, yielding a hybrid SA, in order to obtain good quality networks designs. Additionally, four variants of this algorithm based on different cooling schemes are introduced and analyzed. A broad experimentation using different benchmark networks is carried out to test our proposals. Moreover, a comparison with an approach from the literature reveals the goodness to solve this network design problem.
\end{abstract}

Keywords: Water Distribution Network Design · Optimization • Metaheuristic $\cdot$ Simulated Annealing

\section{Introduction}

A water distribution network consists of thousands of nodes with nonlinear hydraulic behaviour, linked by thousands of interconnecting links. The inherent problem associated with cost optimisation in the design of water distribution networks is due to the nonlinear relationship between flow and head loss and the discrete nature of pipe sizes. As a consequence, the solution concerning the layout, design, and operation of the network of pipes should result from good planning and management procedures. In this way, this problem known as Water Distribution Network Design (WDND) requires to manage an important number of variables (pipes, pipe diameters, demand nodes, water pressure, reservoirs, etc.), and constraints (water velocity, pressure, etc.). This problem, even for simple networks, is very difficult to solve, in particular it is classified as NP-hard [1].

Early research works in the WDND optimization area were focused on the single-period, single-objective, gravity-fed design optimization problem.

(C) Springer Nature Switzerland AG 2019

P. Pesado and C. Aciti (Eds.): CACIC 2018, CCIS 995, pp. 3-16, 2019.

https://doi.org/10.1007/978-3-030-20787-8_1 
The first research works applied linear programming [2,3], and non-linear programming $[4,5]$. After that, the metaheuristics have been used to solve these problems, such as the trajectory-based ones: Simulated Annealing [6,7] and Tabu Search [8]. Also population-based metaheuristics were applied, for example, Ant Colony Optimization [9], Ant Systems [10], Genetic Algorithms [11-13] Scatter Search [14], and Differential Evolution [15].

Recently, the single-period problem was extended to a multi-period setting in which time varying demand patterns occur. Farmani et al. [16] formulated the design problem as a multi-objective optimization problem and apply a multiobjective evolutionary algorithm. In [12], a Genetic Algorithm was used to solve six small instances considering velocity constraint on the water flowing through the distribution pipes. This constraint was also taken into account in [17], but the authors used mathematical programming on bigger, closer-to-reality instances. A Differential Evolution (DE) algorithm was proposed in [18] to minimize the cost of the water distribution network. Another version of a DE algorithm to solve this problem was presented in [19]. An Iterative Local Search [20] was specificallydesigned in order to consider that every demand node has 24 hrs water demand pattern and a new constraint, which imposes a limit on the maximal velocity of water through the pipes.

Based on the problem formulation given by De Corte and Sörensen [20], we propose an optimization technique, based on Simulated Annealing (SA), in order to improve and optimize the distribution network design. Thus, this SA is designed to obtain the optimal type of pipe connecting the supply, demand, and junction nodes in the distribution network. This proposal incorporates a local search procedure in order to improve the layout of the network, arising the Hybrid Simulated Annealing (HSA). The HSA's performance is compared with algorithms present in the literature. This work constitutes an extension of a previous work [21] and includes new content regarding an study and analysis of the main control parameter of the HSA, known as temperature. Moreover, we introduce and statistically compare four HSA's variants, taking into account different schemes to schedule the cooling process. We test the performance of our proposals with a set of networks with different sizes expressed by number of pipes and characteristics. The evaluation considers relevant aspects such as efficiency and internal behavior.

The rest of this article is organized as follows. In Sect. 2, we introduce the problem definition. Section 3 explains our algorithmic proposal, HSA, to solve the WDND optimization problem and the four HSA's variants. Section 4 describes the experimental analysis and the methodology used. Then, we analyze the results obtained by the variants and compare with the obtained by the ILS [20] in Sects. 5 and 6, respectively. Finally, we present our principal conclusions and future research lines.

\section{Multi-Period Water Distribution Network Design}

The objective of the WDND problem is to minimize the total investment cost (TIC) in a water distribution network design. The problem can be characterized 
as: simple-objective, multi-period, and gravity-fed. Two restrictions are considered: the limit of water speed in each pipe and the demand pattern that varies in time. The network can be modeled by a connected graph, which is described by a set of nodes $N=\left\{n_{1}, n_{2}, \ldots\right\}$, a set of pipes $P=\left\{p_{1}, p_{2}, \ldots\right\}$, a set of loops $L=\left\{l_{1}, l_{2}, \ldots\right\}$, and a set of commercially available pipe types $T=\left\{t_{1}, t_{2}, \ldots\right\}$. The TIC is obtained by the formula shown in Eq. 1,

$$
\min T I C=\sum_{p \in P} \sum_{t \in T} L_{p} I C_{t} x_{p, t}
$$

where $I C_{t}$ is the cost of a pipe $p$ of type $t, L_{p}$ is the length of the tube, and $x_{p, t}$ is the binary decision variable that determines whether the tube $p$ is of type $t$ or not. The objective function is limited by: physical laws of mass and energy conservation, minimum pressure demand in the nodes, and the maximum speed in the pipes, for each time $\tau \in \mathcal{T}$. These laws are explained in the following paragraphs.

Mass Conservation Law: It must be satisfied for each node $N$ in each period of time $\tau$. This law establishes that the volume of water flowing towards a node in a unit of time must be equal to the flow that leaves it (see Eq. 2),

$$
\sum_{n_{1} \in N / n} Q_{\left(n_{1}, n\right), \tau}-\sum_{n_{2} \in N / n} Q_{\left(n, n_{2}\right), \tau}=W D_{n, \tau}-W S_{n, \tau} \quad \forall n \in N \quad \forall \tau \in \mathcal{T}
$$

where $Q_{\left(n_{1}, n\right), \tau}$ is the flow from node $n_{1}$ to node $n$ at time $\tau, W S_{n, \tau}$ is the external water supplied and $W D_{n, \tau}$ is the external water demanded.

Energy Conservation Law: It states that the sum of pressure drops in a closed circuit in an instant of time $\tau$ is zero. These drops can be approximated using the Hazen-Williams equations with the parameters used in EPANET 2.0 [22] (the hydraulic solver used in this paper), as indicated in Eq. 3.

$$
\sum_{p \in l}\left[\frac{10.6668 y_{p, \tau} Q_{p, \tau}^{1.852} L_{p}}{\sum_{t \in T}\left(x_{p, t} C_{t}^{1.852} D_{t}^{4.871}\right.}\right]=0 \quad \forall l \in L \quad \forall \tau \in \mathcal{T}
$$

In Eq. 3, $y_{p, \tau}$ is the sign of $Q_{p, \tau}$ that indicates changes in the water flow direction relative to the defined flow directions, $Q_{p, \tau}$ is the amount of water flowing through pipe $p$ in time $\tau, L_{p}$ is the pipe length, $C_{t}$ is the Hazen-Williams roughness coefficient of pipe type $t$, and $D_{t}$ is the diameter of pipe type $t$.

Minimum Pressure Head Requirements: for each node $n$ in each period of time $\tau$, it must be satisfied (see Eq. 4),

$$
H_{n, \tau}^{\min } \leq H_{n, \tau} \quad \forall n \in N \quad \forall \tau \in \mathcal{T}
$$

being $H^{\min }$ the minimum node pressure and $H_{n, \tau}$ the node's current pressure.

Maximum Water Velocity: The water velocity $v_{p, \tau}$ can not exceed the maximum stipulated speed $v_{p, \tau}^{\max }$. Equation 5 shows this relationship.

$$
v_{p, \tau} \leq v_{p, \tau}^{\max } \quad \forall p \in P \quad \forall \tau \in \mathcal{T}
$$


Table 1. Different solutions or network designs in vector representation.

\begin{tabular}{l|l|r|r|r|r|r|r|r|r|l}
\hline \multirow{2}{*}{ Solution } & Pipe ID & 1 & 2 & 3 & 4 & 5 & 6 & 7 & 8 & Feasibility \\
\cline { 2 - 10 } & Length $(\mathrm{m})$ & 31 & 20 & 35 & 37 & 24 & 50 & 12 & 65 & TIC \\
\hline \multirow{2}{*}{1} & Diam. (mm) & 150 & 150 & 80 & 80 & 100 & 60 & 60 & 80 & Feasible \\
\cline { 2 - 10 } & Cost & 1550 & 1000 & 1225 & 1295 & 912 & 1100 & 264 & 2275 & 9621 \\
\hline \multirow{2}{*}{2} & Diam. (mm) & 150 & 150 & 80 & 60 & 100 & 60 & 60 & 80 & Infeasible \\
\cline { 2 - 9 } & Cost & 1550 & 1000 & 1225 & 814 & 912 & 1100 & 264 & 2275 & 9140 \\
\hline
\end{tabular}

\section{Our Proposal for the Multi-Period WDND Problem}

Simulated Annealing (SA) [23], a simple trajectory-based metaheuristic, is based on the principles of statistical thermodynamics, whereby the annealing process requires heating and then slowly cooling the physical material until it solidifies into a perfect crystalline structure. The SA algorithm simulates the energy changes in a system subjected to a cooling process until it converges to an equilibrium state (steady frozen state), where the physical material states correspond to problem solutions, the energy of a state to cost of a solution, and the temperature to a control parameter.

At the beginning (with a high temperature), SA accepts solutions with high cost values under a certain probability in order to explore the search space and to escape from local optima. During the annealing process this probability decreases according to temperature cooling; intensifying the search and reducing the exploration in order to exploit a restricted area of a search space.

Simulated annealing evolves by a sequence of transitions between states and these transitions are generated by transition probabilities. Consequently, SA can be mathematically modeled by Markov chains, where a sequence of chains is generated by a transition probability, which is calculated involving the current temperature.

The proposal consists in adapting and hybridizing the SA algorithm to solve the Multi-Period WDND optimization problem, arising Hybrid Simulated Annealing (HSA) algorithm. A solution to this problem is a network, as shown in Fig. 1(a) and (b). A network or a solution is represented by a vector, where each element is the diameter selected for that pipe, as can be seen in Table 1. In this table the vectors that represent the candidate solutions in Fig. 1(a) and (b) are shown. The total investment cost for each solution is calculated by the Eq. 1, using the input data from tables (c) and (d) of Fig. 1. The first solution is hydraulically feasible (satisfying all constraints mentioned in Sect.2) and the second one is infeasible (violating the minimum pressure constraint in node 7 ).

In Algorithm 1, we show a pseudo-code of the HSA algorithm to solve the WDND optimization problem. HSA uses the EPANET 2.0 toolkit [22] to solve the hydraulic equations, since this hydraulic solver is applied in most existing works. HSA generates a feasible initial solution $S_{0}$ applying both HighCost and Lowcost mechanisms proposed in [20] (line 2). After the evaluation of the initial 

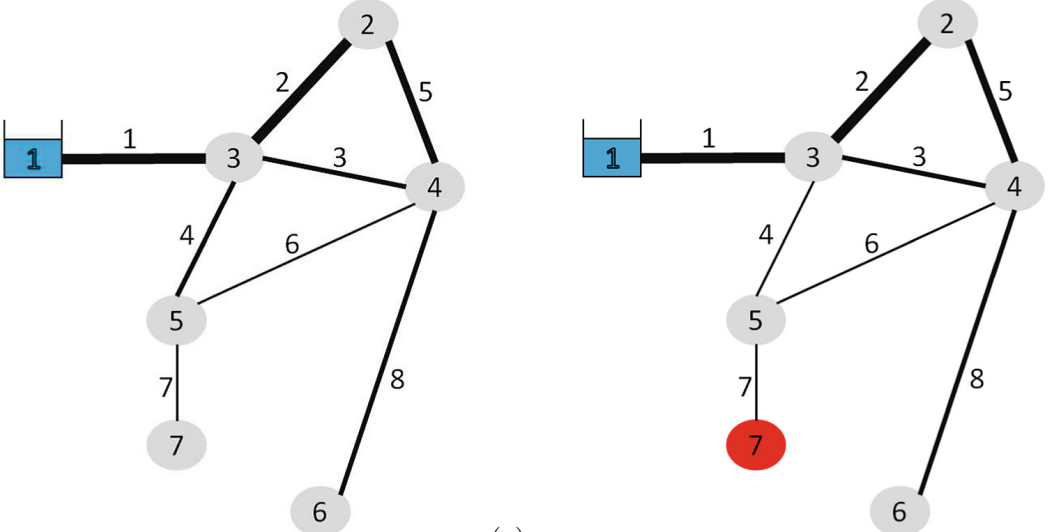

(a)

\begin{tabular}{cc}
\hline Pipe ID Length $\mathbf{( m )}$ \\
\hline 1 & 31 \\
2 & 20 \\
3 & 35 \\
4 & 37 \\
5 & 24 \\
6 & 50 \\
7 & 12 \\
8 & 65 \\
\hline
\end{tabular}

\begin{tabular}{ccc}
\hline $\begin{array}{c}\text { Diam. Roughness } \\
(\mathbf{m m})\end{array}$ & $\begin{array}{c}\text { unitless } \\
\text { Cost }\end{array}$ \\
\hline 60 & 130 & 22 \\
80 & 130 & 35 \\
100 & 130 & 38 \\
150 & 130 & 50 \\
200 & 130 & 61 \\
\hline & $(\mathrm{d})$ &
\end{tabular}

(c)

Fig. 1. Different solutions or network designs. (a) Solution 1; (b) solution 2; (c) pipe lengths; (d) available pipe types with their corresponding costs.

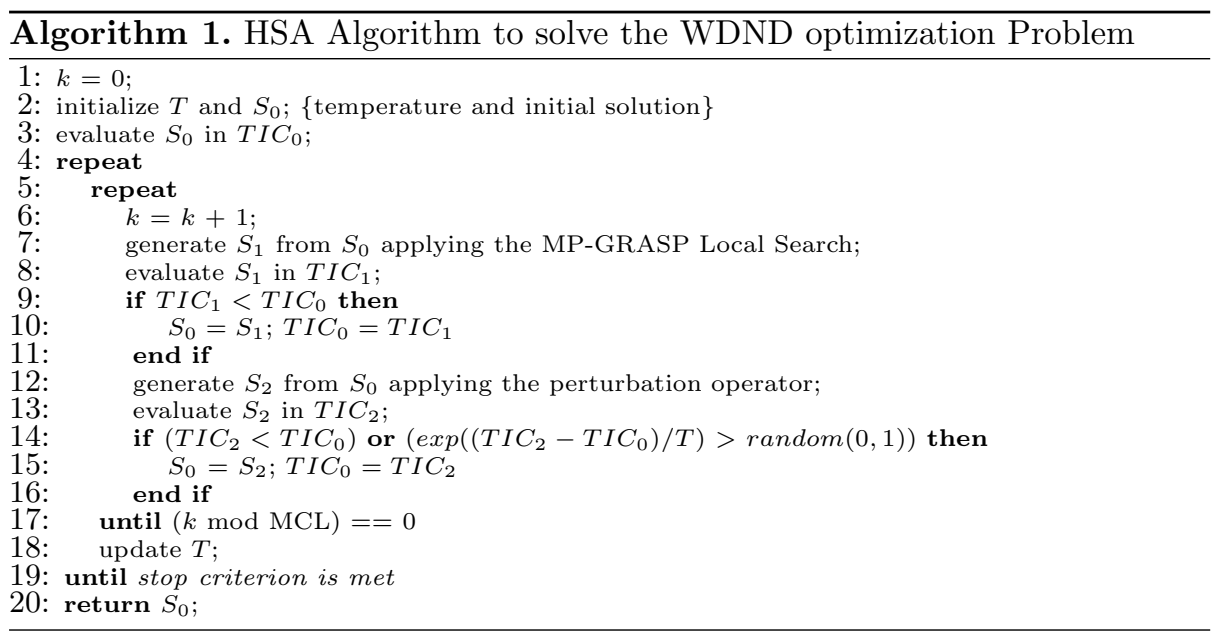


solution (line 3), an iterative process starts (lines 4 to 19). As a first step in the iteration, the hybridization is carried out in order to intensify the search into the current region of the solution space. In this way a feasible solution, $S_{1}$, is obtained by applying the MP-GRASP local search [20] to $S_{0}$ (line 7 ), and then a greedy selection mechanism is performed (lines 9-11). As a consequence, $S_{0}$ can be replaced by $S_{1}$ if this is better than $S_{0}$. In the next step a perturbation operator is used to obtain a feasible neighbor, $S_{2}$, from $S_{0}$ (line 12), in order to explore another areas of the search space. This perturbation randomly changes some pipe diameters. If $S_{2}$ is worse than $S_{0}, S_{2}$ can be accepted under the Boltzmann probability (line 14, second condition). In this way, at high temperatures $(T)$ the exploration of the search space is strengthened. In contrast, at low temperatures the algorithm only exploits a promising region of the solution space, intensifying the search. In order to update $T$, a cooling schedule [23] is used (line 18) and it is applied after a certain number of iterations $(k)$ given by the Markov Chain Length $(M C L)$ (line 17). Finally, SA ends the search when the total evaluation number is reached or the $T \neq 0$.

Most features in SA, such as search space, perturbation operator, and cost (evaluation) function, are fixed by the problem definition. The only feature that is variable during the process is the temperature. Therefore one of the most important features in simulated annealing is the choice of the annealing schedule, and many attempts have been made to derive or suggest good schedules [24]. In this work, we study the behavior of the most known cooling process in the literature to solve the Multi-Period WDND optimization problem, arising three new HSA variants as explained in the following.

- HSA $A_{\text {Prop }}$ applies the proportional cooling scheme, also called geometric schedule [23], in order to reduce the temperature, as the Eq. 6 shows:

$$
T_{k+1}=\alpha * T_{k}
$$

where $\alpha$ is a constant close to, but smaller than, 1 . Particularly, we calculate $\alpha$ as follows:

$$
\alpha=\frac{k}{k+1}
$$

This scheme is the most popular cooling function because, the temperature decay is not too slow neither too fast allowing to achieve an equilibrium between exploitation and exploration.

- HS $A_{E x p}$ uses the exponential cooling scheme [23] to produce the temperature decay. The Eq. 8 describes these process, where the constant $\alpha^{k}<1$ is calculated in the Eq. 9. This schedule quickly cools the temperature reducing the required time and iterations to converge to a good solution. In big and complex problems, this becomes in a disadvantage, given that the equilibrium between the exploitation and exploration is broken.

$$
\begin{gathered}
T_{k+1}=T_{k} * \alpha^{k} \\
\alpha^{k}=\frac{e^{k}}{e^{1+k}}
\end{gathered}
$$


Table 2. Information on the HydroGen networks.

\begin{tabular}{l|l|l|l|l|l|l|l|l|l}
\hline Network & $\begin{array}{l}\text { Meshedness } \\
\text { coefficient }\end{array}$ & Pipes & $\begin{array}{l}\text { Demand } \\
\text { nodes }\end{array}$ & $\begin{array}{l}\text { Water } \\
\text { reservoirs }\end{array}$ & Network & $\begin{array}{l}\text { Meshedness } \\
\text { coefficient }\end{array}$ & Pipes & $\begin{array}{l}\text { Demand } \\
\text { nodes }\end{array}$ & $\begin{array}{l}\text { Water } \\
\text { reservoirs }\end{array}$ \\
\hline HG-MP-1 & 0.2 & 100 & 73 & 1 & HG-MP-9 & 0.1 & 295 & 247 & 2 \\
\hline HG-MP-2 & 0.15 & 100 & 78 & 1 & HG-MP-10 & 0.2 & 397 & 285 & 2 \\
\hline HG-MP-3 & 0.1 & 99 & 83 & 1 & HG-MP-11 & 0.15 & 399 & 308 & 2 \\
\hline HG-MP-4 & 0.2 & 198 & 143 & 1 & HG-MP-12 & 0.1 & 395 & 330 & 3 \\
\hline HG-MP-5 & 0.15 & 200 & 155 & 1 & HG-MP-13 & 0.2 & 498 & 357 & 2 \\
\hline HG-MP-6 & 0.1 & 198 & 166 & 1 & HG-MP-14 & 0.15 & 499 & 385 & 3 \\
\hline HG-MP-7 & 0.2 & 299 & 215 & 2 & HG-MP-15 & 0.1 & 495 & 413 & 3 \\
\hline HG-MP-8 & 0.15 & 300 & 232 & 2 & & & & &
\end{tabular}

- HS $\boldsymbol{A}_{\text {Log }}$ employs the logarithmic cooling scheme [25], which modifies the temperature, as shown in Eq. 10. In this Equation, the chain converges to a global and minimal energy value, where the constant $C$ is computed as the Eq. 11 shows. This schedule is too slow to be applied in practice but has the property of the convergence proof to a global optimum [26].

$$
\begin{aligned}
& T_{k+1}=C * T_{k} \\
& C=\frac{\ln (k)}{\ln (1+k)}
\end{aligned}
$$

Furthermore, we propose a fourth HSA variant, named $\boldsymbol{H} \boldsymbol{S} \boldsymbol{A}_{\boldsymbol{R} \text { and }}$. This new variant combines the three previous explained cooling schemes in only one schedule process. In each iteration, $\mathrm{HSA}_{\text {Rand }}$ randomly selects one of these schemes in order to reduce the temperature. In this way, we try to enhance the HSA by aggregating the advantages of these three schemes and mitigating their disadvantages.

\section{Experimental Design}

In this section, we introduce the experimental design used in this approach, the execution environment, and the result analysis. In order to evaluate HSA, the HydroGen instances of WDND optimization problem [27] are solved. These instances, HG-MP-i, arise from 15 different distribution networks (see Table 2). A set of 16 different pipe types is used and their characteristics and costs can be found in Table 3. The demand nodes are divided into five categories (domestic, industrial, energy, public services, and commercial demand nodes), each one with a corresponding base load and demand pattern ${ }^{1}$. In this way, five different instances are considered for each HG-MP-i network, totalling 75 instances.

The computational environment used in this work to carry out the experimentation consists of computers with INTEL I7 $3770 \mathrm{~K}$ quad-core processors $3.5 \mathrm{GHz}$, 8GB RAM, and the Slackware Linux with 3.2.29 kernel version.

\footnotetext{
${ }^{1}$ The base loads can be found in the EPANET input files of the instances.
} 
Table 3. Available pipe types and their corresponding costs.

\begin{tabular}{l|l|l|l|l|l|l|l}
\hline Number & Diam. (mm) & Roughness & Cost & Number & Diameter & Roughness & Cost \\
\hline 1 & 20 & 130 & 15 & 9 & 200 & 130 & 116 \\
\hline 2 & 30 & 130 & 20 & 10 & 250 & 130 & 150 \\
\hline 3 & 40 & 130 & 25 & 11 & 300 & 130 & 201 \\
\hline 4 & 50 & 130 & 30 & 12 & 350 & 130 & 246 \\
\hline 5 & 60 & 130 & 35 & 13 & 400 & 130 & 290 \\
\hline 6 & 80 & 130 & 38 & 14 & 500 & 130 & 351 \\
\hline 7 & 100 & 130 & 50 & 15 & 600 & 130 & 528 \\
\hline 8 & 150 & 130 & 61 & 16 & 1,000 & 130 & 628 \\
\hline
\end{tabular}

Because of the stochastic nature of the algorithms, we performed 30 independent runs of each instance to gather meaningful experimental data and apply statistical confidence metrics to validate our results and conclusions. As a result, a total of 9000 executions $(75 \times 4 \times 30)$ were carried out. Before performing the statistical tests, we first checked whether the data followed a normal distribution by applying the Shapiro-Wilks test. Where the data was distributed normally, we later applied an ANOVA test. Otherwise, we used the Kruskal-Wallis (KW) test. This statistical study allows us to assess whether or not there were meaningful differences between the compared algorithms with a confidence level of $99 \%$.

\section{Analysis of the Results Obtained by Our Proposals}

In this section, we summarize and analyze the results of using the four proposed HSA's variants $\left(\mathrm{HSA}_{\text {Prop }}, \mathrm{HSA}_{E x p}, \mathrm{HSA}_{L o g}\right.$, and $\mathrm{HSA}_{\text {Rand }}$ ) on all the problem instances, following the next methodology. First, we analyze the behavior of these variants considering the results shown in the Table 4 . The columns $2-5$ show the average of the best cost values found by these four variants for the 75 instances grouped by their corresponding distribution network. The minimal cost values found by each group are boldfaced. In the last column, the results of the Kruskall-Wallis test are summarized, where the symbol "+" indicates that the behavior of the four HSA's variants are statistically similar, while the symbol "-" specifies that these behaviors are significantly different. Secondly, we analyze the temperature decay for each proposed HSA taking into account the variation of the temperature parameter during the search, as shown in the Fig. 2.

Regarding the quality point of view, $\mathrm{HSA}_{\text {Prop }}$ finds the best cost in many more instances than the rest of variants, i.e. HSA Prop achieves the best solutions in eight instances, followed by $\mathrm{HSA}_{\text {Exp }}$ with three, $\mathrm{HSA}_{\text {Rand }}$ with two, and $\mathrm{HSA}_{\text {Log }}$ with only one instance (see the boldfaced values in the Table 4). These differences between the behaviors are supported by the KW results, which indicate that the cooling schemes drive the search in significant different ways, 
Table 4. Averages of the best cost values found by each proposed variant, which are grouped by network.

\begin{tabular}{l|r|r|r|r|l}
\hline Network & \multicolumn{1}{|c|}{ HSA $_{\text {Prop }}$} & \multicolumn{1}{|c|}{$\mathrm{HSA}_{E x p}$} & \multicolumn{1}{l}{$\mathrm{HSA}_{\text {Log }}$} & \multicolumn{1}{c}{$\mathrm{HSA}_{\text {Rand }}$} & $\mathrm{KW}$ \\
\hline HG-MP-1 & $\mathbf{3 3 5 8 8 2 , 8 0}$ & 338817,60 & 338223,00 & 338250,60 & - \\
\hline HG-MP-2 & 298842,40 & 304934,80 & 298651,80 & $\mathbf{2 9 7 2 4 1 , 6 0}$ & - \\
\hline HG-MP-3 & 387088,80 & $\mathbf{3 7 1 4 9 0 , 4 0}$ & 386142,80 & 386133,60 & - \\
\hline HG-MP-4 & $\mathbf{6 9 0 0 3 3 , 4 0}$ & 698058,60 & 691564,60 & 697496,40 & - \\
\hline HG-MP-5 & 722217,60 & 722878,00 & $\mathbf{7 1 8 7 5 4 , 8 0}$ & 724301,00 & - \\
\hline HG-MP-6 & $\mathbf{7 4 1 6 3 8 , 2 0}$ & 747825,80 & 751994,80 & 742786,60 & - \\
\hline HG-MP-7 & 817261,80 & 825763,40 & 831011,80 & $\mathbf{8 1 2 4 2 8 , 2 0}$ & - \\
\hline HG-MP-8 & $\mathbf{8 5 5 4 7 8 , 6 0}$ & 862003,00 & 867300,00 & 866820,20 & - \\
\hline HG-MP-9 & $\mathbf{8 3 4 8 2 1 , 4 0}$ & 841512,20 & 842372,60 & 845829,40 & - \\
\hline HG-MP-10 & $\mathbf{7 8 8 4 2 2 , 2 0}$ & 796010,80 & 801549,60 & 809821,60 & - \\
\hline HG-MP-11 & $\mathbf{9 0 9 0 3 7 , 8 0}$ & 915537,80 & 915997,20 & 912114,80 & - \\
\hline HG-MP-12 & 1046426,00 & $\mathbf{1 0 4 3 7 2 8 , 2 0}$ & 1054274,40 & 1058030,60 & - \\
\hline HG-MP-13 & $\mathbf{1 7 9 5 6 8 , 0 0}$ & 1196634,00 & 1190972,00 & 1189460,00 & - \\
\hline HG-MP-14 & $\mathbf{1 0 8 5 1 9 4 , 8 0}$ & 1092782,40 & 1087786,20 & 1087889,40 & - \\
\hline HG-MP-15 & 1161200,00 & $\mathbf{1 1 5 5 2 5 4 , 0 0}$ & 1166694,00 & 1170664,00 & - \\
\hline
\end{tabular}

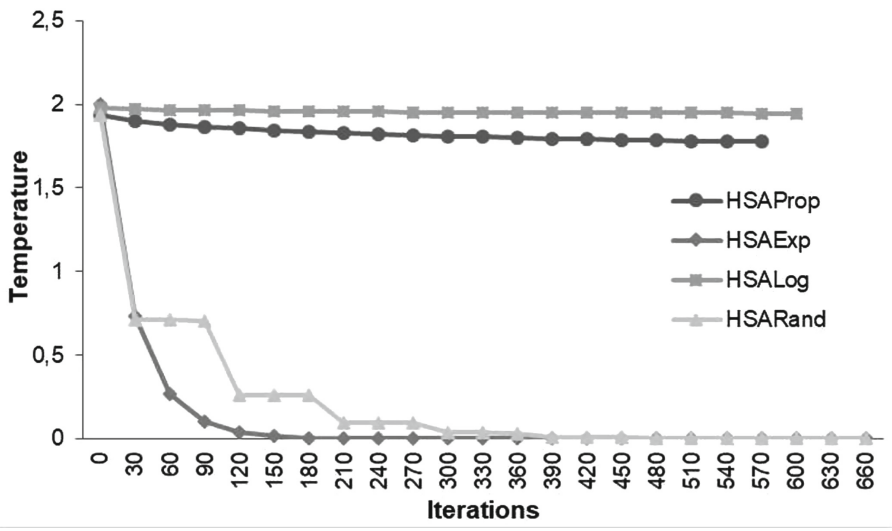

Fig. 2. Average temperature decay during the search.

strengthening the search when the proportional scheme is used. This scheme produces a temperature decay that allow an adequate exploration at the beginning, enabling a greater exploitation at the ending of the search.

If the Fig. 2 is analyzed, no quick temperature convergence to zero is observed when $\mathrm{HSA}_{\text {Prop }}$ and $\mathrm{HSA}_{L o g}$ are executed, but the temperature decay is greater in the first one. This last property is the reason of the HSA Prop's success to find the minimal cost values in more than $50 \%$ of the instances. Conversely, regarding 
Table 5. Average of the best TIC values obtained by ILS and HSA Prop for all instances grouped by the corresponding network. The best values are boldfaced.

\begin{tabular}{l|r|l|l|r|r}
\hline Network & \multicolumn{1}{|l|}{ ILS } & HSA $_{\text {Prop }}$ & Network & \multicolumn{1}{|l}{ ILS } & HSA Prop \\
\hline HG-MP-1 & 339200 & $\mathbf{3 3 5 8 8 3}$ & HG-MP-9 & $\mathbf{8 3 1 2 0 0}$ & 834821 \\
\hline HG-MP-2 & 303800 & $\mathbf{2 9 8 8 4 2}$ & HG-MP-10 & 790600 & $\mathbf{7 8 8 4 2 2}$ \\
\hline HG-MP-3 & 389200 & $\mathbf{3 8 7 0 8 9}$ & HG-MP-11 & $\mathbf{9 0 7 0 0 0}$ & 909038 \\
\hline HG-MP-4 & 694200 & $\mathbf{6 9 0 0 3 3}$ & HG-MP-12 & 1022800 & 1046426 \\
\hline HG-MP-5 & 728200 & $\mathbf{7 2 2 2 1 8}$ & HG-MP-13 & 1190400 & $\mathbf{1 1 7 9 5 6 8}$ \\
\hline HG-MP-6 & 750400 & $\mathbf{7 4 1 6 3 8}$ & HG-MP-14 & $\mathbf{1 0 7 0 2 0 0}$ & 1085195 \\
\hline HG-MP-7 & 818000 & $\mathbf{8 1 7 2 6 2}$ & HG-MP-15 & $\mathbf{1 1 2 2 6 0 0}$ & 1161200 \\
\hline HG-MP-8 & $\mathbf{8 5 1 6 0 0}$ & 855479 & & & \\
\hline
\end{tabular}

$\mathrm{HSA}_{\text {Exp }}$ and $\mathrm{HSA}_{\text {Rand }}$, the temperature converges quickly to values close to zero restricting the exploration at the beginning of the search, although $\mathrm{HSA}_{\text {Exp }}$ outperforms $\mathrm{HSA}_{\text {Rand }}$ when the solution quality is analysed. It is remarkable that, the stop condition is achieved by all HSA's variants when the number of evaluations (EPANET calls) is equal to 1,500,000 but the temperature remains greater than zero.

Summarizing, the proportional cooling scheme allows HSA Prop $_{\text {outperforms }}$ the remaining HSA's variants, by balancing the exploitation and exploration during the search. As a consequence, $\mathrm{HSA}_{\text {Prop }}$ obtains the best networks designs doing the same computational effort than the others variants.

\section{Comparison of $\mathrm{HSA}_{\text {Prop }}$ and the Literature Approaches}

Regarding that $\mathrm{HSA}_{\text {Prop }}$ obtained the best results, we select this variant to compare its performance with the ILS proposed in [20]. This metaheuristic is chosen from literature for this comparison, since its authors also used the HydroGen instances to test it. In this way, our results can be compared with ones of the state-of-the-art, allowing to know the level of quality reached by our proposal.

The methodology used to analyze the results is described in the following. First, we study the HSA behavior comparing the best cost values found by HSA $_{\text {Prop }}$ and ILS [20] for the 75 instances, grouped by their corresponding distribution network, as presented in the Table 5. Secondly, we analyze the HSA Prop convergence, in comparison with ILS, taking into account the cost values found at the $1 \mathrm{e}+05,3 \mathrm{e}+05,5 \mathrm{e}+05,10 \mathrm{e}+05$, and 15e+05 EPANET calls (evaluations), as shown in the Fig. 3. Besides, the HSA's execution times (in seconds) to carry out the maximum number of evaluations for each test case, grouped by network, are shown in the Fig. 4. Note that, we only present the HSA's total execution time for all test cases, because no data about this metric are reported by De Corte and Sörensen in [20]. 
Analyzing the Table 5, we detect that $\mathrm{HSA}_{\text {Prop }}$ finds solutions with less TIC values than the ones of ILS in nine networks. As a consequence, in $60 \%$ of the problem instances better cost values are found when they are solved by HSA Prop. The HSA advantage arises out of the Boltzmann probability application to accept high TIC values, which allows to diversify the search to escape from local optima.

From the convergence point of view, we observe that HSA Prop finds solutions with TIC values near to the best ones in $80 \%$ of the instances, with only $1 \mathrm{e}+05$ evaluations. Instead, ILS needs at least $3 \mathrm{e}+05$ evaluations for that, besides this is achieved in only $66 \%$ of the test cases.
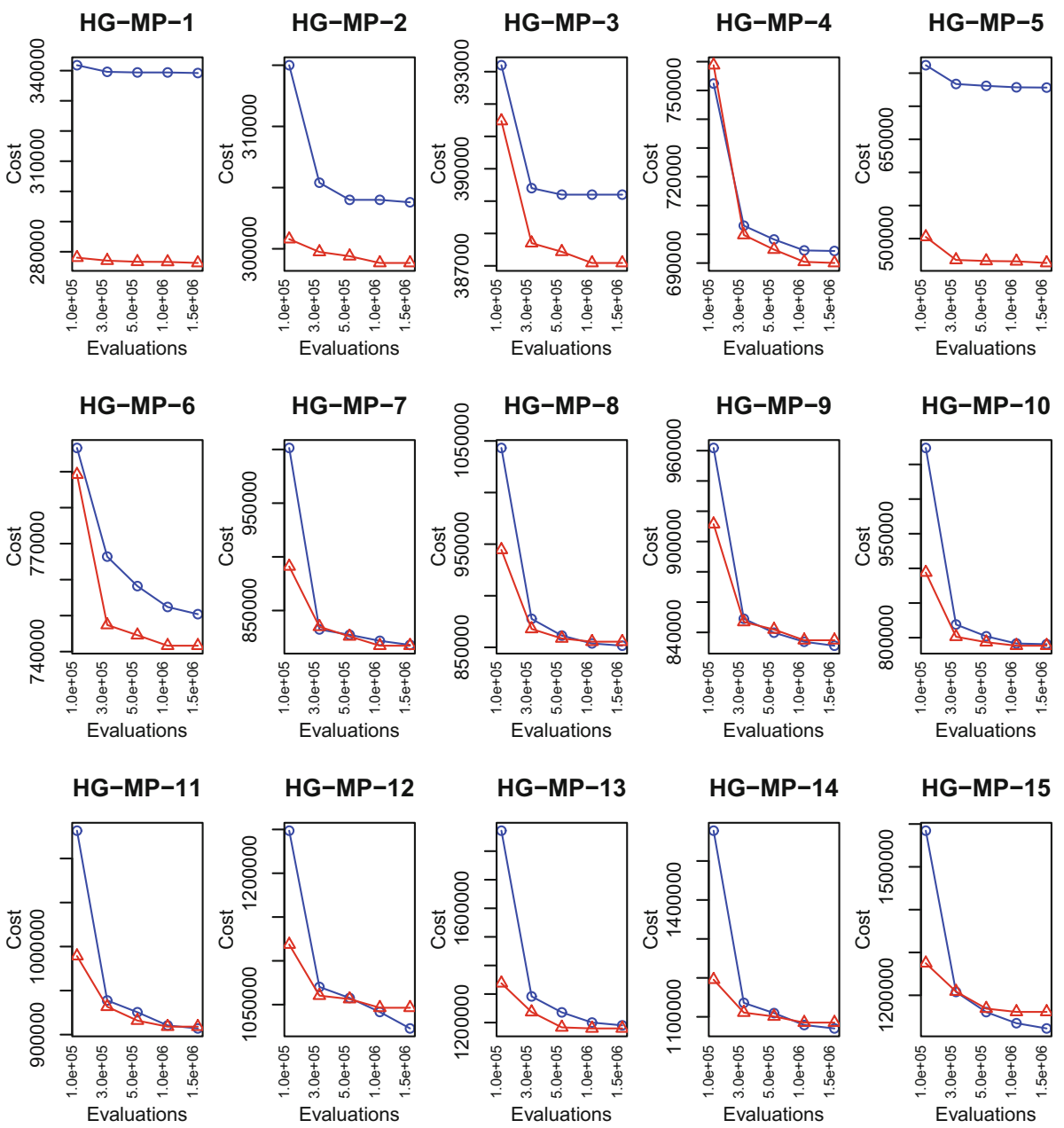

HG-MP-15

ILS

$\mathrm{HSA}_{\text {Prop }}$

Fig. 3. Evolution of the TIC values during the search for all instances. 


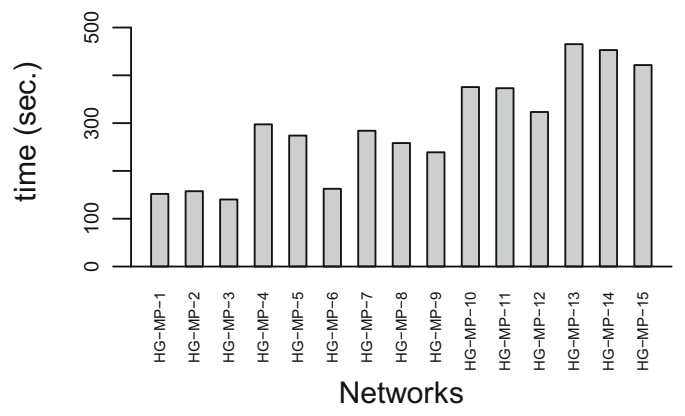

Fig. 4. Average of the total $\mathrm{HSA}_{\text {Prop }}$ execution times for all instances grouped by the corresponding network.

Evaluating both the Fig. 4 and the Table 2 together, we notice that the HSA's execution time is affected by the number of pipes and demand nodes. In this way, five groups of three networks can be formed exhibiting similar execution times. These instances have consecutive numbers, e.g. the set of the HG-MP-1, 2, and 3 networks have similar number of pipes and demand nodes, and so on. Being the set formed by the HG-MP-13, 14, and 15 networks the most expensive cases to solve. Furthermore, analyzing what happened into each set of networks, the network with more demand nodes consumes less execution time than the other two, since more feasible solutions exist and HSA needs less time to find one of them.

Summarizing, HSA $_{\text {Prop }}$ outperforms ILS for the $60 \%$ of the problem instances when the result quality is considered. Moreover, a quick convergence to good solutions is also evidenced by our proposal in most of the problem instances. Furthermore, the HSA's runtime is affected by the growing and combination of the number of pipes and demand nodes.

\section{Conclusions}

In this paper, we have proposed water distribution network designs considering the multi-period settings with time varying demand patterns. The technique proposed to obtain these network designs is a hybrid Simulated Annealing algorithm, called HSA, which solves the hydraulic equations by using the EPANET 2.0 toolkit. HSA combines an WDND-adapted SA with the MPGRASP local search [20]. Furthermore, four different HSA's variants (HSA Prop, $\mathrm{HSA}_{E x p}, \mathrm{HSA}_{L o g}$, and $\mathrm{HSA}_{\text {Rand }}$ ) have been created by applying the proportional, exponential, logarithmic, and random cooling schemes respectively. For this study, we have tested 75 instances that come from 15 different HydroGen networks.

An important point is that all the proposed HSA's variants had an equivalent computational effort, because all of them carried out the same number of evaluations. In this context $\mathrm{HSA}_{\text {Prop }}$, the algorithm that uses the proportional 
cooling scheme to reduce the temperature, found the best networks designs outperforming the remaining proposals.

Moreover, $\mathrm{HSA}_{\text {Prop }}$ 's results were compared with the obtained by the ILS proposed in [20] to solve this problem. As a consequence, we observed that this HSA variant also outperformed the results obtained by ILS in more than half $(60 \%)$ of instances. Additionally, HSA achieved a better exploration than ILS, because of the Boltzmann probability application to accept solutions that can explore new areas of the search space. This advantage combined with the local search allowed HSA to converge quickly on the best solutions.

For future works, we will improve the HSA to solve the multi-period WDND optimization problem, by introducing changes in the initialization method of the temperature. We are also interested in testing larger dimension instances, as close as possible to real scenarios.

Acknowledgments. The authors acknowledge the support of Universidad Nacional de La Pampa and the Incentive Program from MINCyT. The second author is also funded by CONICET.

\section{References}

1. Yates, D.F., Templeman, A.B., Boffey, T.B.: The computational complexity of the problem of determining least capital cost designs for water supply networks. Eng. Optim. 7(2), 143-155 (1984)

2. Alperovits, A., Shamir, U.: Design of optimal water distribution systems. Water Resour. Res. 13(6), 885-900 (1977)

3. Kessler, A., Shamir, U.: Analysis of the linear programming gradient method for optimal design of water supply networks. Water Resour. Res. 25(7), 1469-1480 (1989)

4. Fujiwara, O., Khang, D.: A two-phase decomposition method for optimal design of looped water distribution networks. Water Resour. Res. 26(4), 539-549 (1990)

5. Duan, N., Mays, L.W., Lansey, K.E.: Optimal reliability-based design of pumping and distribution systems. J. Hydraul. Eng. 116(2), 249-268 (1990)

6. Loganathan, G., Greene, J., Ahn, T.: Design heuristic for globally minimum cost water-distribution systems. J. Water Res. Plan. Manag. 121(2), 182-192 (1995)

7. da Conceicao Cunha, M., Sousa, J.: Hydraulic infrastructures design using simulated annealing. J. Infrastruct. Syst. 7(1), 32-39 (2001)

8. da Conceicao Cunha, M., Ribeiro, L.: Tabu search algorithms for water network optimization. Eur. J. Oper. Res. 157(3), 746-758 (2004)

9. Maier, H.R., et al.: Ant colony optimization for design of water distribution systems. J. Water Resour. Plan. Manag. 129(3), 200-209 (2003)

10. Zecchin, A.C., Simpson, A.R., Maier, H.R., Nixon, J.B.: Parametric study for an ant algorithm applied to water distribution system optimization. IEEE Transact. Evol. Comput. 9(2), 175-191 (2005)

11. Dandy, G.C., Simpson, A.R., Murphy, L.J.: An improved genetic algorithm for pipe network optimization. Water Resour. Res. 32(2), 449-458 (1996)

12. Gupta, I., Gupta, A., Khanna, P.: Genetic algorithm for optimization of water distribution systems. Environ. Model. Softw. 14(5), 437-446 (1999) 
13. Bi, W., Dandy, G.C., Maier, H.R.: Improved genetic algorithm optimization of water distribution system design by incorporating domain knowledge. Environ. Model. Softw. 69, 370-381 (2015)

14. Lin, M.-D., Liu, Y.-H., Liu, G.-F., Chu, C.-W.: Scatter search heuristic for leastcost design of water distribution networks. Eng. Optim. 39(7), 857-876 (2007)

15. Vasan, A., Simonovic, S.P.: Optimization of water distribution network design using differential evolution. J. Water Resour. Plan. Manag. 136(2), 279-287 (2010)

16. Farmani, R., Walters, G.A., Savic, D.A.: Trade-off between total cost and reliability for anytown water distribution network. J. Water Resour. Plan. Manag. 131(3), 161-171 (2005)

17. Bragalli, C., D'Ambrosio, C., Lee, J., Lodi, A., Toth, P.: On the optimal design of water distribution networks: a practical MINLP approach. Optim. Eng. 13(2), 219-246 (2012)

18. Uma, R.: Optimal design of water distribution network using differential evolution. Int. J. Sci. Res. (IJSR) 5(11), 1515-1520 (2016)

19. Mansouri, R., Mohamadizadeh, M.: Optimal design of water distribution system using central force optimization and differential evolution. Int. J. Optim. Civil Eng. 7(3), 469-491 (2017). http://ijoce.iust.ac.ir/article-1-310-en.html

20. De Corte, A., Sörensen, K.: An iterated local search algorithm for water distribution network design optimization. Network 67(3), 187-198 (2016)

21. Bermudez, C.A., Minetti, G.F., Salto, C.: SA to optimize the multi-period water distribution network design. In: XXIX Congreso Argentino de Ciencias de la Computación, CACIC 2018, pp. 12-21 (2018)

22. Rossman, L.A.: The EPANET Programmer's Toolkit for Analysis of Water Distribution Systems (1999)

23. Kirkpatrick, S., Jr, C.G., Vecchi, M.: Optimization by simulated annealing. Science 220, 671-680 (1983)

24. Talbi, E.-G.: Metaheuristics: From Design to Implementation. Wiley, Hoboken (2009)

25. Hajek, B.: Cooling schedules for optimal annealing. Math. Oper. Res. 13(2), 311329 (1988)

26. Geman, S., Geman, D.: Stochastic relaxation, Gibbs distributions, and the Bayesian restoration of images. IEEE Trans. Pattern Anal. Mach. Intell. 6(6), 721-741 (1984). https://doi.org/10.1109/TPAMI.1984.4767596

27. De Corte, A., Sörensen, K.: Hydrogen. http://antor.uantwerpen.be/hydrogen. Accessed on 27 June 2018 